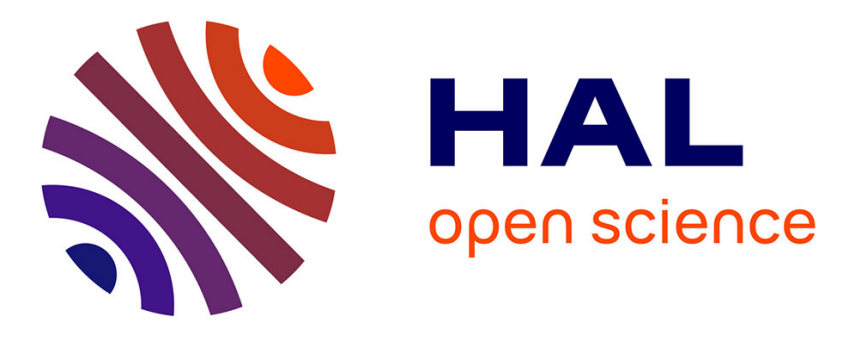

\title{
Drop impact on superhydrophobic surface with protrusions
}

Xiaofeng Jiang, Enle Xu, Guoguang Wu, Huai-Zhi Li

\section{To cite this version:}

Xiaofeng Jiang, Enle Xu, Guoguang Wu, Huai-Zhi Li. Drop impact on superhydrophobic surface with protrusions. Chemical Engineering Science, 2020, 212, pp.115351. 10.1016/j.ces.2019.115351. hal-02447383

\section{HAL Id: hal-02447383 \\ https://hal.science/hal-02447383}

Submitted on 21 Dec 2021

HAL is a multi-disciplinary open access archive for the deposit and dissemination of scientific research documents, whether they are published or not. The documents may come from teaching and research institutions in France or abroad, or from public or private research centers.
L'archive ouverte pluridisciplinaire HAL, est destinée au dépôt et à la diffusion de documents scientifiques de niveau recherche, publiés ou non, émanant des établissements d'enseignement et de recherche français ou étrangers, des laboratoires publics ou privés.

\section{(ㄷ)(1) $\$$}

Distributed under a Creative Commons Attribution - NonCommercial| 4.0 International 


\title{
Drop impact on superhydrophobic surface with protrusions
}

\author{
Xiaofeng Jiang ${ }^{\mathrm{a}, \mathrm{b}}$, Enle $\mathrm{Xu}^{\mathrm{a}}$, Guoguang $\mathrm{Wu}^{\mathrm{a}}$, Huai Z. Li ${ }^{\mathrm{b}} *$ \\ ${ }^{a}$ School of Chemical Engineering and Technology, China University of Mining and Technology, \\ Xuzhou 221116, PR China \\ ${ }^{\mathrm{b}}$ Laboratory of Reactions and Process Engineering, CNRS, University of Lorraine, 1, Rue \\ Grandville, BP 20451, 54001 Nancy cedex, France
}

Keywords: drop impact, superhydrophobic, protrusion, deformation, breakup

\begin{abstract}
The phenomenon of drop impact is of significant importance to many engineering applications. In this work, water drops impact on superhydrophobic surfaces with or without protrusions were experimentally investigated under relatively low We numbers, using a high-speed camera. The focus was given to the spreading, bouncing and breakup behaviors on three different protrusions (semicircle, equilateral triangle and square). Our results show that the presence of protrusions not only alters the impact dynamics but also drastically affect the drop deformation. It was found that water drops persist the memory of the protrusions' geometry during the rebounding process and the hierarchical structure of protrusion surfaces leads drops to bounce off and break up more easily. A dimensionless analysis reveals that the protrusion with different geometries distinctly enhances the expanding process and reduces the contact time compared to the planar surface.
\end{abstract}




\section{Introduction}

Many engineering applications involve liquid impacting and wetting on solid surface, for example trickle bed reactors, surface coatings, inkjet printing and so on (Lunkad et al., 2007; Bhat et al., 2019; Arogeti et al., 2019). In these processes, the impact of liquid (mostly in the form of drops) with solid surface plays a crucial role for the liquid-solid contact, especially for the later momentum / heat / mass transfer. However, the property of solid surface largely affects the drop impact behavior (Cottin-Bizonne et al., 2003). Nowadays, liquid drop impacting on superhydrophobic surfaces attracts a lot of interest in both industrial applications and academic research due to its wide applications, such as drag reduction (Cottin-Bizonne et al., 2003), waterproof and self-cleaning (George et al., 2016; Chen et al., 2016; Zhang et al., 2016), inkjet printing (van Dam and Le Clerc, 2004), spray coating (Sampath and Jiang, 2001), anti-icing (Wang et al., 2015), pesticide deposition (Bergeron et al., 2000), soil erosion (Wijewardana et al., 2016), etc. A liquid drop impacting on superhydrophobic surface usually induces short-term spreading, splashing, receding and bouncing (Yarin, 2006) and these post-impact dynamics significantly depend on liquid initial conditions (density, viscosity and velocity), surface properties (roughness, geometry and mechanical strength) and solid-liquid interactions (wettability). For example, drops by adding a small amount of polymer can inhibit the bouncing (Bergeron et al., 2000) and soft substrates can suppress the splashing (Pepper et al., 2008). A lot of previous literature have been devoted to drop impact on superhydrophobic plane surfaces with nano-/micro- structures (Leclear et al., 2016; 
Lei et al., 2014; Lv et al., 2016; Malla et al., 2017; Patil et al., 2016). However, to our best knowledge, very few attention was given to the drop impact on superhydrophobic surfaces with macrostructures which is comparable to the drop size.

Macrostructures on solid surfaces are ubiquitous in our daily life, for example, ridges, posts and other roughness from mechanical defects, aging defects or natural structure on plant leaves, etc. Recently, some research reveals that macrostructures, in the range of $100 \mu \mathrm{m}-1 \mathrm{~mm}$, can generate new dynamic behaviors of drops, like millimetric crenellations trapping the Leidenfrost drops (Dupeux et al., 2011), millimetric grooves guiding drops motion (Mertaniemi et al., 2011) or cutting drops (Yanashima et al., 2012) etc. More importantly, these submillimeter-scale macrostructures exert a pronounced influence on the liquid-solid contact time, compared to the drop impact on superhydrophobic surface with nano-/microstructures. For example, Bird et al. (2013) demonstrated that the contact time was reduced by $\sim 37 \%$ through adding spacing sub-millimeter-scale ridges with a triangle section on the superhydrophobic surface, where the ridges break the drop into two. Liu et al. (2014) reported the pancake bouncing of drops on superhydrophobic surface patterned with lattices of sub-millimeter-scale tapered posts decorated with nanostructures. This kind of bouncing leads drops to rebound without retraction so that the contact time could be reduced fourfold compared to the conventional bouncing. Shen et al. (2015) investigated the impact behavior of drops on superhydrophobic surface with macrostructure of rational equilateral triangle cylinders and demonstrated the limited overall contact time of approximately $5.5 \mathrm{~ms}$. 
Gauthier et al. (2015) designed experiments of water drops impacting on superhydrophobic wires and found that the contact time was reduced by a square root of subunits compared to the regular repellent surface. Recently, Song et al. (2017) fabricated macro-aniso-superhydrophobic surfaces with different wire spacing and found the contact time was remarkably reduced with a flying-eagle bouncing. The above-mentioned studies demonstrated that the macrostructure could reduce the contact time by either modifying the bouncing behavior or enhancing the breakup behavior, but the effect of a single macrostructure on drop impact deformation and dynamics has not been well understood.

The macrostructures especially protrusions in real applications or academic research are more or less with arc, slope or angle shape. Here, we choose three typical stripy protrusion geometries with arc, slope and right-angle section to be the models of actual defects. The three protrusions share the same base dimension, while the cross-section shape varies, i.e. semicircle, equilateral triangle and square. Our previous work (Jiang and Li, 2017) about liquid drop hurdling over barriers indicates that the presence of protrusions can greatly affect the drop motion and deformation. We numbers $\left(W \mathrm{e}=\rho d u^{2} / \sigma\right)$ stands for the ratio of kinetic energy to surface energy. Here, $\rho, d, u$, and $\sigma$ are the density, equivalent diameter, impact velocity and surface tension of the liquid drop, respectively. Considering the high We numbers leading to more deviations or difficulties due to the surface slip, splashing, ramified fluid body or disintegrations (Song et al., 2017; Zhang et al., 2014), thus our focus here was given to the water drop impact on superhydrophobic surfaces with protrusions of 
various geometries (semicircle, equilateral triangle and square), under relatively low We numbers.

\section{Experimental section}

A schema of experimental setup was designed and displayed in Fig. 1. The stainless steel nozzle I (inner diameter $d^{\prime}=0.6 \mathrm{~mm}$ and outside diameter $d^{\prime \prime}=0.97$ $\mathrm{mm}$ ) and nozzle II (inner diameter $d^{\prime}=1.8 \mathrm{~mm}$ and outside diameter $d^{\prime \prime}=2.5 \mathrm{~mm}$ ) together with a syringe pump (Harvard Apparatus, USA) were used to generate drops of volume: $V=16 \mu \mathrm{L}$ and $V=37 \mu \mathrm{L}$ (corresponding to a diameter $\sim 3.14 \mathrm{~mm}$ and $\sim 4.14 \mathrm{~mm}$, respectively). The drop must remain larger than the protrusion, and smaller than the capillary length. Then the equivalent diameter $d$ of liquid drop was obtained from the drop volume resulting from the ratio between the liquid flowrate through the nozzle and the drop formation frequency, its error was less than $2 \%$. Water (density $\rho=1000 \mathrm{~kg} / \mathrm{m}^{3}$, viscosity $\eta=1 \mathrm{mPa} . \mathrm{s}$, surface tension $\sigma=72 \mathrm{mN} / \mathrm{m}$ ) was the work fluid. Aluminum was the material that we adopted to produce the basic surfaces and three geometries - semicircle, equilateral triangle, and square, shown in the right-bottom of Fig. 1, were prepared as protrusions. These protrusion surfaces were fabricated through precise milling. The superhydrophobic surface, both the substrate and protrusions, were treated with the commercial product NeverWet $^{\mathrm{TM}}$ (Rust-Oleum Corporation, USA) consisting of superhydrophobic nanoparticles dispersed in volatile solvents. After a thin polymer coating, both the substrate and the protrusions share the similarly superhydrophobic properties with the apparent contact 
angle over $160^{\circ}$ for pure water drops. During the drop motion, the advancing and receding angles are $161 \pm 2^{\circ}$ and $146 \pm 2^{\circ}$, respectively. The preparation and treatment method of protrusion surfaces is the same with our previous work (Jiang and $\mathrm{Li}, 2017)$. Considering the persistence of superhydrophobicity, all the tests were conducted under the same pressure and temperature within one or two days. In addition, Weber number in our experiments is relatively low by controlling the release height of the drop, so the mechanical damage to the superhydrophobic surface is very small. In spite of this, the superhydrophobicity of both the surfaces and protrusions were regularly verified through the contact angle measurement and renewed if diminution observed.

The drop impact velocity and resulting Weber number were varied by adjusting the release height of the drop through a linear displacement system. The drop impact velocity was directly monitored by the displacement of the drop in time by the high-speed camera. The drop impact velocity and Weber number were repeatable with $<3 \%$ and $<10 \%$ errors, respectively. The length of base surface substituted by protrusions in the direction of drop motion is defined as $W$. Here, $W=1 \mathrm{~mm}$ or $W=$ $2 \mathrm{~mm}$, which is in the same order of magnitude with drops but smaller than the drops' diameter. Drop impact behavior was recorded with a Phantom v7.11 camera (Vision Research, USA). The visualization speed remained 8000 frames per second (fps) and the exposure time was $5 \mu \mathrm{s}$. The obtained images were analyzed frame by frame through the self-programmed Matlab. It is worth mentioning that the symmetry of drops impact is firstly ensured by the coaxial nozzle with protrusions (Fig. 1) and then 
checked by the liquid distribution, for example the drop volume distribution by two sides of protrusions during spreading or breakup.

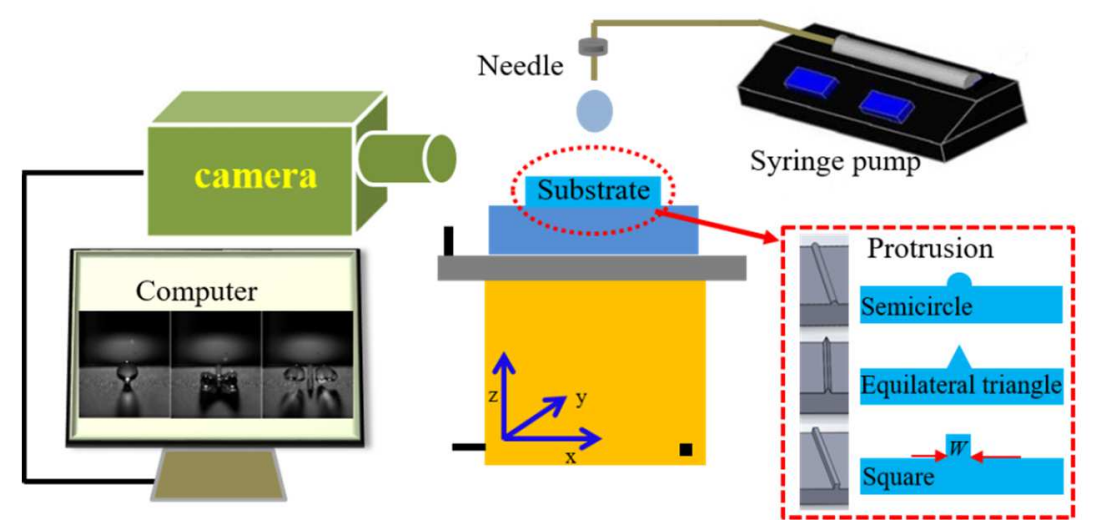

Fig. 1. Experimental setup for liquid drops impact on superhydrophobic surfaces with various protrusions. The cross-section shapes of protrusions are semicircle, equilateral triangle and square, respectively.

\section{Results and discussion}

When a drop impacts on superhydrophobic surface, different shapes may arise depending on the interaction dynamics occurring at the liquid-solid interface. Once a drop contacts with the surface, inertial force causes the drop to spread in the form of surface wave. When it reaches the maximum spreading diameter, kinetic energy is partly transformed into surface energy and subsequently, the drop retracts. If the dissipated energy within the droplet and friction with the surface is sufficiently small, it will still have enough kinetic energy to rebound. Weber number stands for the ratio of kinetic energy to surface energy. For smaller We numbers, drops are ellipsoidal during the contact and the deformation is small (Richard and Quéré, 2000; Clanet et al.,2004). For larger We numbers, drop deforms dramatically and the shape is quite 
different. Here, we analyze the morphologic evolution of drops impact on protrusion superhydrophobic surfaces under relatively lower and higher We numbers, respectively.

Snapshots of water drops gently impacting on superhydrophobic surfaces are shown in Fig. 2. In Fig. 2a, a descendent water drop approaches the plane surface and once it lands on, the front part continues to expand. With the liquid spreading, the drop increases in width and decreases in height. After it reaches the maximum spreading, i.e. the maximum lateral extension, the drop begins to retract, deforms and finally detaches from the substrate. When the plane surface is incorporated with single semicircle, equilateral triangle and square protrusion, the descendent water drop first touches the zenith of protrusion and then begins to spread. Due to different geometries of protrusions, the spreading mode varies. Drops can easily spread along the curvature of the semicircle protrusion (Fig. 2b) or the slope of the triangle protrusion (Fig. 2c) and no gap can be observed between drops and surfaces. However, the square protrusion (Fig. 2d) due to its larger height and larger cross-section at the top of the protrusion, intercepts the middle part of the drop liquid and leads the liquid to expand from two sides. The two-ear shape with visible slots can be seen at $t=6.5 \mathrm{~ms}$ in Fig. 2d. With time evolution, the drops all spread out on the surface and evolve into a seemingly flat surface in the third images of Fig. $2 b, 2 c$ and $2 d$. Then, the middle part on protrusions sinks to be an arc though a big slot can be observed at $t=20.3 \mathrm{~ms}$ in Fig. 2d. After the maximum spreading, drops begin to retract and a small bulge forms in the center, except the square protrusion where the drop gradually becomes a flat 
surface. That could stem from the repellency of different geometries at the top: both the semicircle and triangle protrusion exert a repellent force from a zenith point with an arc or taper angle, while the square protrusion repels the drop by a plane surface. Zenith point of semicircle and triangle protrusion exerts a very large repellent force, which naturally results in a small bulge in the center part of the drop. However, the uniform repellent force from the top of square protrusion leads the drop to retract without a small bulge in the middle. The small bulges in Fig. $2 b$ and $2 c$ rapidly grow up and two sides continue to retract, leading to an arch shape. The arches continue to deform and eventually rebound to leave the solid surfaces. However, in Fig. 2d, two ends with the flat top surface firstly form a 'flyover' and then a 'minus' over the square protrusion due to the repellent force from both the lateral sides of square protrusion and the bottom substrate. The 'minus' retracts and deforms but it doesn't rebound to leave the square protrusion due to the deficiency of initial kinetic energy. Normally, if the drop impact velocity is large enough, drops will rebound from the protrusion surfaces. Here, drops can rebound from the semicircle and triangle protrusion but not on the square protrusion, which is maybe due to the large interception surface at the top as well as the larger height of square protrusion, or the larger energy dissipation with a higher contact surface. In conclusion, the geometry difference of protrusions makes drops' deformation quite specific. And it was found that drops remain nearly the same shape with protrusions at the moment that they leave the surface. 


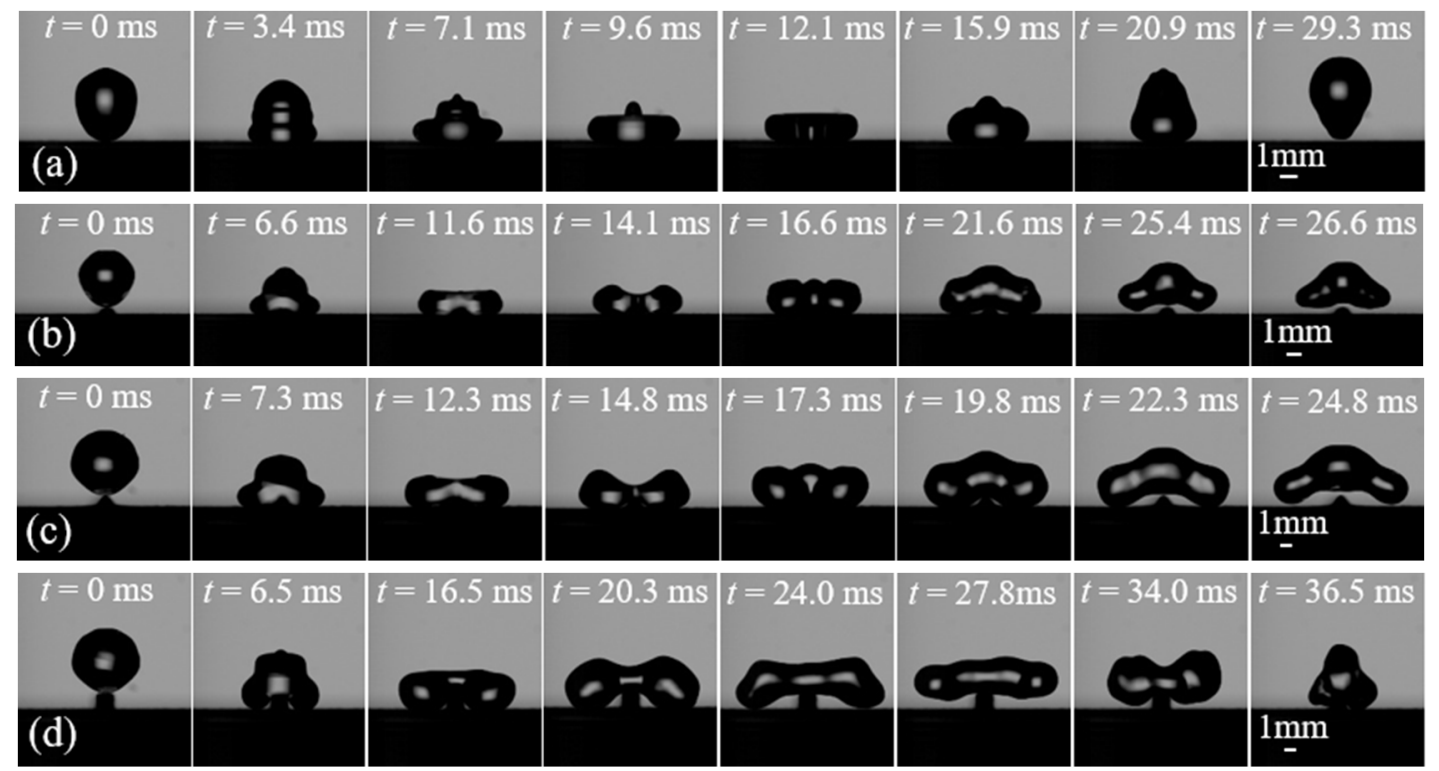

Fig. 2. Gentle impact of water drops $(V=37 \mu \mathrm{L})$ on superhydrophobic surfaces with or without protrusions. (a) Plane surface $(W e=6.8)$; (b) Semicircle protrusion (We $=$ 7.9); (c) Triangle protrusion (We $=7.1)$; (d) Square protrusion $(W e=7.1)$.

Fig. 3 shows the relatively violent impact situation. When We number increases, water drop hits the plane surface and the larger inertial force leads the front part to stretch while the upper part of the free surface deforms as the kinetic energy is converted into surface energy and viscous dissipation (Fig. 3a). Similarly, the drop rapidly reaches its maximum diameter and then rebounds in the way of a jet. The top part of the drop pinches off and the rest liquid continues to leave the plane surface and deforms itself. In Fig. 3b, the drop spreads outward and the center sinks to be an arc on the semicircle protrusion. The liquid around the semicircle is then rejected to be a semicircle shape and the thread connecting the two sides becomes thinner and thinner. Finally, the mother drop breaks up into two partitions through the thread pinch-off. Similarly, the drop spreading on the triangle protrusion (Fig. 3c) also sinks in the center. Two small slots occur due to the water-repellency from two slopes $(\tau=11.5$ 
ms indicated in Fig. 3c). Then the knife-like ridge cuts the thin layer to form two droplets. However, in Fig. 3d, a water drop spreads to both sides and the middle liquid is jacked up by the square protrusion. When the drop reaches the maximum spreading, the two ends grow up and the middle liquid leaves the square protrusion, forming a square boundary. The two ends continuously expand outward while the middle liquid thins to be a thread. At one time the thread pinches off.

From the inclined images (insert in third images of Fig. 3a, b, c and d), liquid drop remains a round shape on the planar surface with the center submerged and edge raised, while drops spreading on three protrusions take on a four-corner-welling-up shape, somewhat like a butterfly. The four-corner-welling-up shape was naturally generated by the protrusions at higher We number, which was also reported in the previous works (Bird et al., 2013; Gauthier et al., 2015). In fact, the presence of protrusions leads liquid drops to easily expand to two sides due to the nonuniform height, and the liquid on the top surface of the protrusions expands slowly, which causes the asymmetric drop spreading with liquid accumulation at the four corners. The strip geometry of protrusions bifurcates the drop liquid at expanding stage, and after the maximum extension, four lobes merge into two subunits on two sides of the protrusions. Interestingly, these two subunits connected by a thread persist the memory of the protrusions' geometry during the rebounding process. This is mainly from the repellent force of superhydrophobic coating on three geometries, i.e. semicircle protrusion repels drop with the semispherical surface, triangle protrusion with the inclined surfaces and square protrusion with three planar surfaces. The drop 
on both sides of the protrusions evolves into a large subunit and eventually breaks up through the thread. Within our investigated experimental range, we found all breakup leads drops to fragment into two droplets. More videos could be seen from the supplementary materials (Video S1-S3).

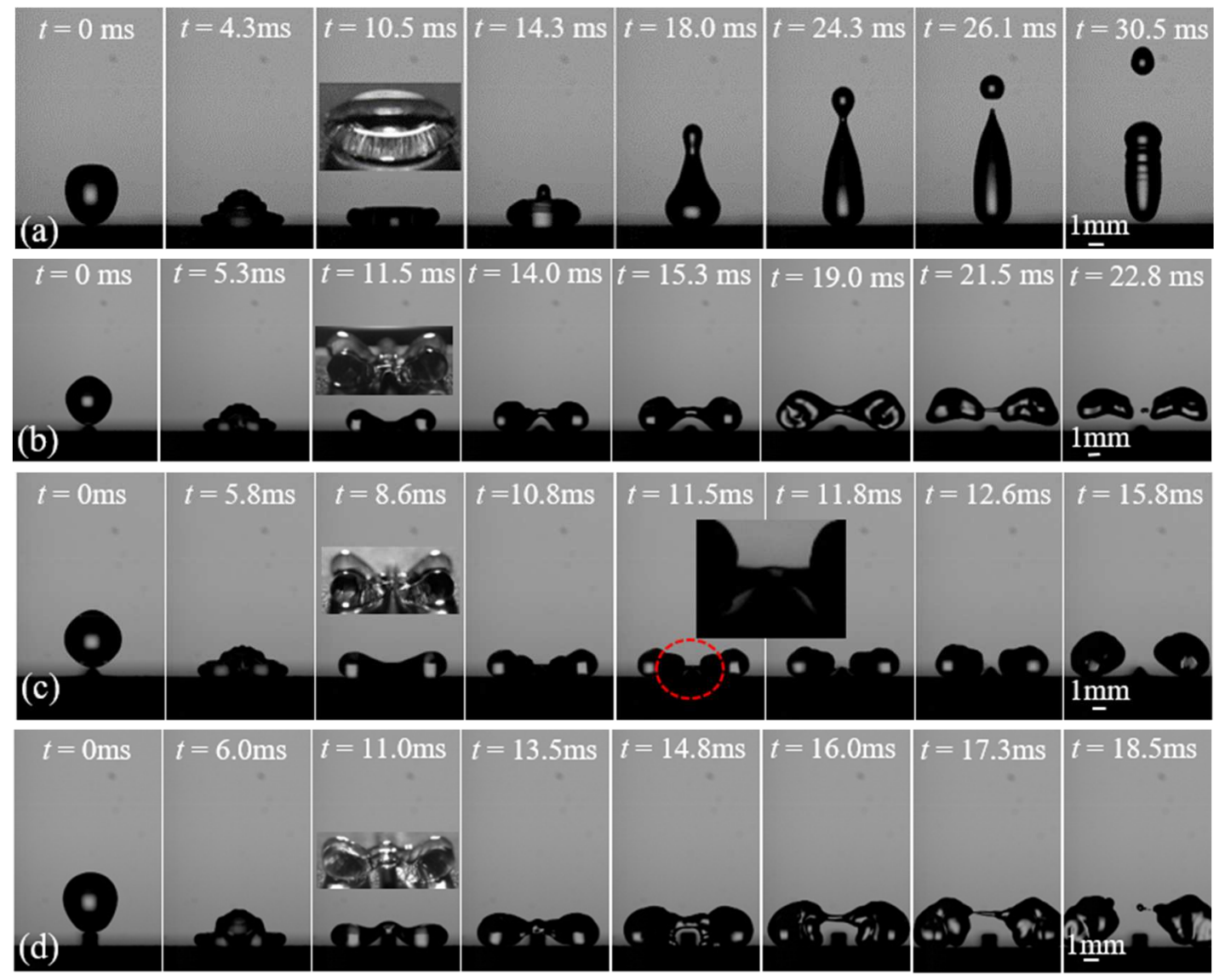

Fig. 3. Relatively violent impact of water drops $(V=37 \mu \mathrm{L})$ on the superhydrophobic surfaces with or without protrusions. (a) Plane surface (We = 21.7); (b) Semicircle protrusion (We $=19.4)$; (c) Triangle protrusion (We =19.1); (d) Square protrusion $(W e=20.8)$.

From the above-mentioned two situations, the presence of protrusions not only enhances the shape deformation but also alters the impact dynamics. No matter under lower or higher We numbers, drops remain the memory of protrusions' geometry during the rebounding process and this memory effect is more pronounced at higher 
We numbers. The flat section of the square protrusion leads to more complex and special deformation compared to semicircle and triangle protrusions. At lower We numbers, drops tends to bounce several times without breakup, while at higher We numbers, drops pinch off through the thread connecting two subunits. The presence of protrusions redistributes the liquid mass and leads drops to break up in horizontal direction, which is quite different from the vertical breakup of their counterpart without protrusions. Fig. 4 illustrates the transition from non-breakup to breakup pattern of water drops impact on protrusions within our investigated ranges. Obviously, the threshold value of We number for water drops breakup is in the order: plane $>$ semicircle $>$ triangle $>$ square. It can be concluded that the presence of protrusions can enhance the breakup and the triangle and square protrusions show great cutting effect.

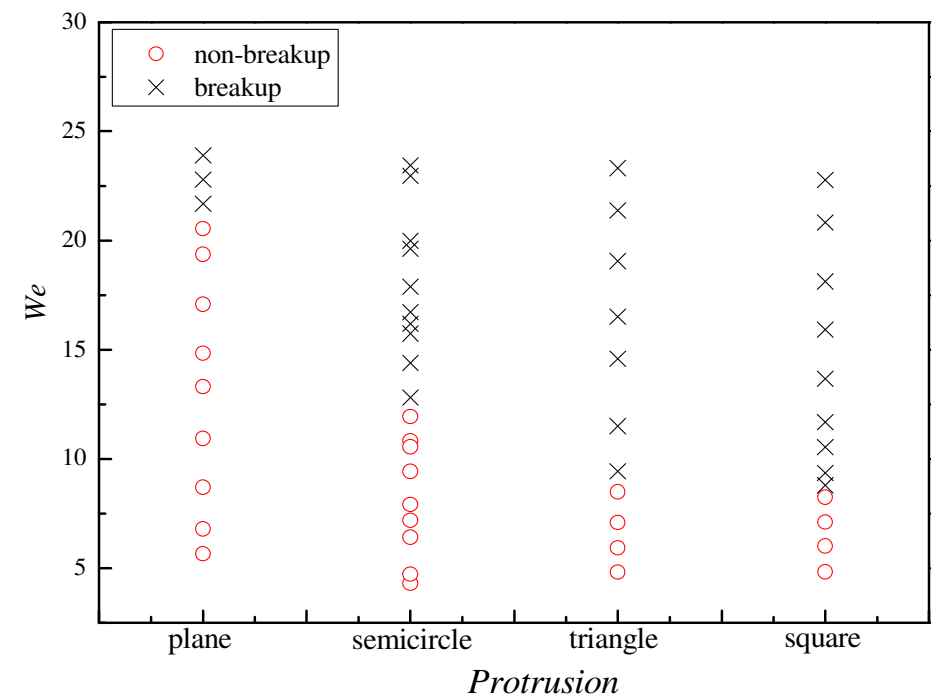

Fig. 4. Occurrence of breakup of water drops impact on each test surfaces as a function of We number. This figure only stands for the occurrence, rather than drop breakup mechanism. 
To quantitatively investigate the drop impact behavior, we mainly analyze the spreading diameter and contact time. Considering the slot between the drop and protrusion, here we define the spreading diameter $D(t)$ as the horizontal width of drops during expanding stage instead of the contact line between drop and solid surface. Thus the evolution of spreading diameter scaled by the initial diameter for drops is given in Fig. 5, at corresponding We numbers of Fig. 2 and Fig. 3, respectively. Due to the slight discrepancy of We numbers, as well as the presence of protrusions, the initial spreading process differs among four kind of surfaces. No matter at low or high We numbers in Fig. 5, drops impact on protrusion surfaces expand wider than that on plane surface and among three protrusions, the square protrusion expands the widest, followed by triangle and then semicircle protrusions. This may be explained from Fig. 5c that the presence of protrusions leads liquid drop to expand more easily outward to two sides, i.e. asymmetric spreading, and the larger the height of protrusion, the wider the expanding due to the larger potential energy. On the other hand, the radial expansion of liquid drop is related to the repellent force from three geometries illustrated in Fig. 5c. The semicircle protrusion repels liquid drop with the smooth arc surface while the triangle protrusion exerts a strong repellent force from the inclined surfaces. However, the square protrusion leads drop to spread in advance on the large intersecting top surface and then expand outward due to the direct repellent force from two shape angles and lateral sides.

It was observed from Fig. 5 that drops bounce off more quickly and break up earlier with the presence of protrusions. And the time for drops bounce-off and 
breakup are in the order: plane $<$ square $<$ semicircle $<$ triangle. In fact, the inclined view in the supplementary materials (Video S1-S3) shows that the liquid retracts faster on the top of protrusions than in other directions, and this asymmetric drop retraction leads drop to bounce off and breakup faster in comparison to the planar surface (Bird et al., 2013). On the other hand, the geometries of protrusions take effect during the force competition. The square protrusion acts as a two-tier plane-plane hierarchical structure; semicircle protrusion is a two-tier arc-plane hierarchical structure; triangle protrusion then is a two-tier corner-plane hierarchical structure. As the free drop flow impacts vertically, the corner-plane hierarchical structure exerts a stronger repellent force to the drop, which leads the drop to quickly bounce off or breakup. In addition, drops contact the square protrusion by a curved surface to a plane surface, instead of a curved surface to a line for the semicircle and triangle protrusions, which makes drops spread on the first-tier and second-tier plane surface successively, so that the breakup time of drops impact on the square protrusion is longer compared to that on the other two protrusions. However, the two-tier plane-plane hierarchical structure of square protrusion leads drops to break up more easily compared to the plane surface due to the strong repellency from the angles and the two vertical sides (Fig. 5c). More importantly, it was found that the breakup of drops on protrusion surfaces is no longer in vertical direction, and breakup occurs either before or after detaching from the protrusion surfaces. This proves that the presence of protrusion greatly affects the impact dynamics. For asymmetric drop 
retraction and breakup, 3D characterization of the drop shape would be required in further investigations.
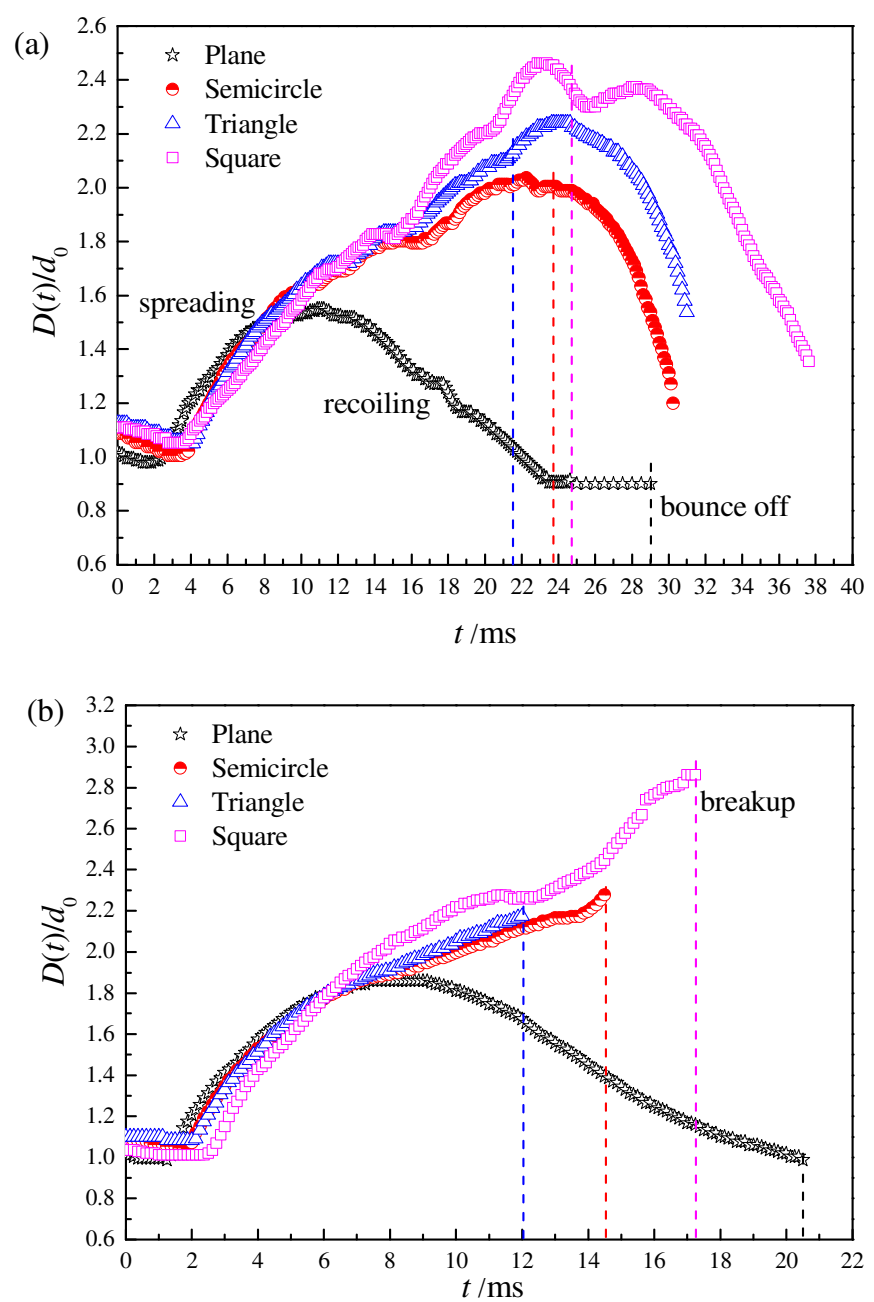

(c)
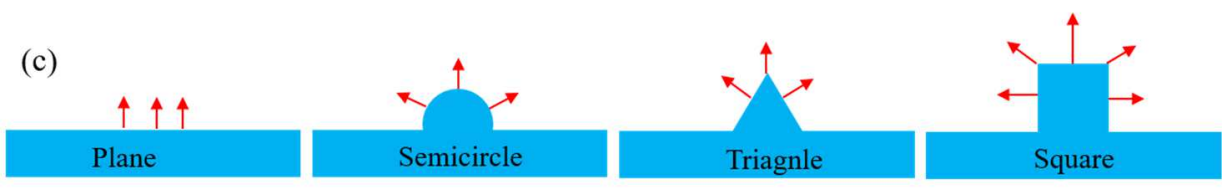

Fig. 5. Dynamics of water drops impacting on the superhydrophobic surfaces with different protrusions under lower We number (a) and higher We number (b) corresponding to the above Fig. 2 and Fig. 3, respectively. The volume of water drops is $V=37 \mu \mathrm{L}$. The dashed line in (a) stands for the time of drops leaving the surface. The dashed line in (b) refers to the time of drops breaking up at each sequence. (c) referring to the analyses model for the repellent force from plane and protrusion surfaces. 
We define a drop contact time $\tau_{c}$ as the time between the first contact and last leave on the solid surface and the maximum spreading diameter $D_{\max }$ as the maximum width of drop in each sequence. Each sequence was analyzed through a self-programmed Matlab procedure and the corresponding maximum spreading diameter $D_{\max }$ could be obtained through the instantaneous widths of drops during impact. Fig. $6 \mathrm{a}$ and $6 \mathrm{~b}$ present the maximum spreading ratio $D_{\max } / d_{0}$ and dimensionless contact time normalized by the inertial time $\tau=\tau_{C} / \sqrt{\rho d_{0}^{3} / \sigma}$ as a function of We number. However, the maximum spreading ratio $D_{\max } / d_{0}$ and dimensionless contact time $\tau$ are not so distinct among the semicircle, equilateral triangle and square protrusions, a more distinct difference could be observed between protrusion surfaces and plane surface. According to the work of Clanet et al. (2004), the maximum spreading ratio $D_{\max } / d_{0}$ could be scaled as $W e^{1 / 4}$ for a low-viscosity drop impact on the non-wettable surface, due to the balance between capillarity and effective acceleration created at the impact. Here, in Fig. 6a, we found the exponent of drops impact on plane superhydrophobic surface is 0.20 , which is quite close to $1 / 4$. This small difference may be issued from the surface structure as well as the experimental conditions. But the exponent of drops impact on protrusion superhydrophobic surfaces is 0.35 , which is larger than that of plane surface. Though the effect of protrusion shape on the exponent value is not so distinct, the larger exponent of drops impact on protrusion surfaces implies that the scaling exponent is sensitive to the surface protrusion. On one hand, the presence of protrusions leads an 
asymmetric spreading, i.e. the liquid drop spreads larger to two sides of protrusions than along the strip of protrusions. Thus liquid drop spreads wider on the protrusion surface than that on the planar surface. On the other hand, the two-tier hierarchical structure of protrusion surfaces leads drops to easily expand to two sides due to the potential energy as well as the repellency from protrusions. Thus, it could be concluded that the presence of protrusions alters the drop spreading dynamics and enhanced the expanding process.

The contact time of drops impact on protrusion surfaces is experimentally found to be less than that of drops impact on plane surface. Except the very small We numbers, the contact time nearly remains the same. The average contact time of water drops impact on plane surface is $18.2 \mathrm{~s}$ for $V=16 \mu \mathrm{L}$ and $28.6 \mathrm{~s}$ for $V=37 \mu \mathrm{L}$. The contact time is a little different from literatures which reports the contact time about 12-14 ms (Wang et al., 2007; Tsai et al., 2009), this can be explained that the experimental conditions are different such as the drop size or We number. While the average contact time of water drops impacting on surfaces with semicircle / triangle / square protrusions is $13.7 \mathrm{~s}$ for $V=16 \mu \mathrm{L}$ and $21.5 \mathrm{~s}$ for $V=37 \mu \mathrm{L}$. From Fig. $6 \mathrm{~b}$, the dimensionless time of drops impacting on plane surface is constant with the value of 0.88 , while that on the protrusion surface is about 0.65 . Thus, the protrusion surfaces indeed reduce the contact time compared to the plane surface. It is worth noting that the contact time of drops impact on the protrusion surface under extremely small We number is much higher than the later constant value. That is maybe because the lower impact velocity with lower initial kinetic energy cannot lead drops to rebound higher, 
especially higher than the protrusions' height, which leads to the longer contact time. In all, the quantitative analyses in Fig. $6 a$ and $6 \mathrm{~b}$ demonstrate that the protrusion surfaces indeed enhance the expanding and reduce the contact time. This may throw some insight into the potential mechanisms involving in practical applications like spray coating, anti-icing, self-cleaning etc.
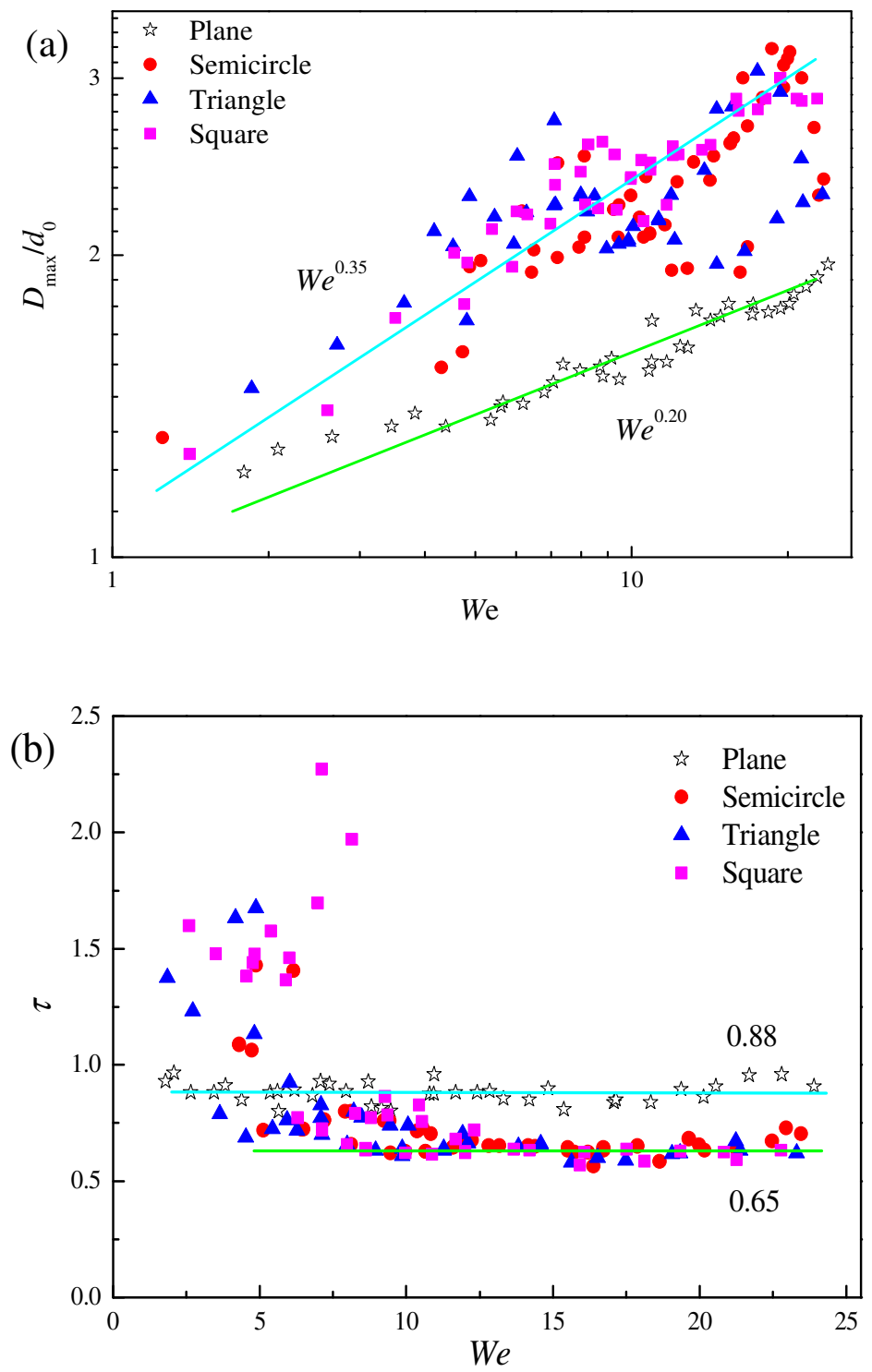

Fig. 6. Quantitative description of water drops impact on the plane surface and protrusion surfaces. (a) Maximum spreading ratio $\left(D_{\max } / d_{0}\right)$ vs. We number. (b) Dimensionless contact time $\tau$ vs. We number. 


\section{Conclusions}

The impact behavior of water drops was investigated on various protrusion surfaces with a high-speed camera. The experimental results reveal that compared to the plane surface, the protrusion surfaces largely enhance the drop deformation and also facilitate the drop breakup. This is attributed to the hierarchical structure of protrusions which redistributes the liquid mass and leads drops to break up in horizontal direction. And it was found that drops persist the memory of protrusions' geometry during the rebounding process. The quantitative analyses demonstrate that the protrusion surfaces indeed reduce the contact time and enhance the expanding process. Although the geometry effect of protrusions has a limited influence on the maximum spreading ratio and dimensionless contact time, it has a significant effect on the morphology evolution. Our observations throw insight into the dynamics of liquid drops impacting on superhydrophobic solid surfaces with various protrusions, which may facilitate the potential applications like anti-icing, coating technology and liquid transportation, etc.

\section{Associated content}


Supporting Information. Dynamical phenomena of $37 \mu \mathrm{L}$ water drops impacting on three protrusions.

\section{Author information}

Corresponding Author

*Email: $\underline{\text { Huai-Zhi.Li@univ-lorraine.fr }}$

\section{Notes}

The authors declare no competing financial interest.

\section{Acknowledgement}

This work is supported by the Fundamental Research Funds for the Central Universities (No. 2019QNA09).

\section{References}

Arogeti, M., Sher, E., Bar-Kohany, T., 2019, Drop impact on small targets with different target-to-drop diameters ratio, Chemical Engineering Science, 193, 89-101.

Bergeron, V., Bonn, D., Martin, J.Y., Vovelle, L., 2000, Controlling droplet deposition with polymer additives. Nature, 405, 772-775.

Bhat, M., Sakthikumar, R., Sivakumar, D., 2019, Fuel drop impact on heated solid surface in film evaporation regime, Chemical Engineering Science, 202, 95-104.

Bird, J.C., Dhiman, R., Kwon, H.M., Varanasi, K.K., 2013, Reducing the contact time of a bouncing drop. Nature, 503, 385-388.

Chen, J., Zhong, X., Lin, J., et al., 2016, The facile preparation of self-cleaning fabrics. Compos. Sci. Technol., 122, 1-9. 
Clanet, C., Béguin, C., Richard, D., Quéré, D., 2004, Maximal deformation of an impacting drop. Journal of Fluid Mechanics, 517, 199-208.

Cottin-Bizonne, C., Barrat, J.L., Bocquet, L., Charlaix, E., 2003, Low-friction flows of liquid at nanopatterned interfaces. Nat. Mater., 2, 237-240.

Dupeux, G., Le Merrer, M., Clanet, C., Quéré, D., 2011, Trapping Leidenfrost drops with crenelations. Phys. Rev. Lett. 107, 114503.

Gauthier, A., Symon, S., Clanet, C., Quéré, D., 2015, Water impacting on superhydrophobic macrotextures. Nat. Commun., 6, 8001.

George, J.E., Rodrigues, V.R.M., Mathur, D., Chidangil, S., George, S.D., 2016, Self-cleaning superhydrophobic surfaces with underwater superaerophobicity. Mater. Design, 100, 8-18.

Jiang, X.F., Li, H.Z., 2017, Liquid drops hurdling barriers of various geometries. Advanced Materials Interfaces, 4, 1700516.

Leclear, S., Leclear, J., Abhijeet, N.V., Park, K.C., Choi, W., 2016, Drop impact on inclined superhydrophobic surfaces. J. Colloid Interf. Sci., 461, 114-21.

Lei, W., Jia, Z.H., He, J.C., Cai, T.M., 2014, Dynamic properties of vibrated drops on a superhydrophobic patterned surface. Appl. Therm. Eng., 62, 507-512.

Liu, Y., Moevius, L., Xu, X., Qian, T., Yeomans, J.M., Wang, Z., 2014, Pancake bouncing on superhydrophobic surfaces. Nature Phys., 10, 515-519.

Lunkad, S.F., Buwa, V.V., Nigam, K.D.P., 2007, Numerical simulations of drop impact and spreading on horizontal and inclined surfaces. Chemical Engineering Science, 62(24), 7214-7224.

Lv, C., Hao, P., Zhang, X , He, F., 2016, Drop impact upon superhydrophobic surfaces with regular and hierarchical roughness. Applied Physics Letters, 108, 141602.

Malla, L.K., Patil, N.D., Bhardwaj, R., Neild, A., 2017, Droplet bouncing and breakup during impact on a microgrooved surface. Langmuir, 33, 9620-9631.

Mertaniemi, H., Jokinen, V., Sainiemi, L., Franssila, S., Marmur, A., Ikkala, O., 2011, Ras, R.H.A., Superhydrophobic tracks for low friction, guided transport of water droplets. Adv. Mater. 23, 2911-2914. 
Patil, N.D., Bhardwaj, R., Sharma, A., 2016, Droplet impact dynamics on micropillared hydrophobic surfaces. Experimental Thermal and Fluid Science, 74, 195206.

Pepper, R.E., Courbin, L., and Stone, H.A., 2008, Splashing on elastic membranes: The importance of early-time dynamics. Phys. Fluids, 20, 082103.

Richard, D., Quéré, D., 2000, Bouncing water drops. Europhys Lett., 50, 769-775.

Sampath, S., Jiang, X., 2001, Splat formation and microstructure development during plasma spraying: deposition temperature effects. Mater. Sci. Eng. A, 304-306, 144-150.

Shen, Y., Tao, J., Tao, H., Chen, S., Pan, L., Wang, T., 2015, Approaching the theoretical contact time of a bouncing droplet on the rational macrostructured superhydrophobic surfaces. Applied Physics Letters, 107, 111604.

Song, M., Liu, Z., Ma, Y., Dong, Z., Wang, Y., Jiang, L., 2017, Reducing the contact time using macro anisotropic superhydrophobic surfaces - effect of parallel wire spacing on the drop impact. NPG Asia Materials, 9, e415.

Tsai, P., Pacheco, S., Pirat, C., Lefferts, L., Lohse, D., 2009, Drop impact upon microand nanostructured superhydrophobic surfaces. Langmuir, 25,12293-12298.

van Dam, D.B., Le Clerc, C., 2004, Experimental study of the impact of an ink-jet printed droplet on a solid substrate. Phys. Fluids, 16, 3403-3414.

Wang, N., Xiong, D.S., Li, M.T., Deng, Y.L., Shi, Y., Wang, K., 2015, Superhydrophobic surface on steel substrate and its anti-icing property in condensing conditions. Appl. Surf. Sci., 355, 226-232.

Wang, Z., Lopez, C., Hirsa, A., Koratkar, N., 2007, Impact dynamics and rebound of water droplets on superhydrophobic carbon nanotube arrays. Applied Physics Letters, 91, 243120.

Wijewardana, N.S., Müller, K., Moldrup, P., Clothier, B., Komatsu, T., Hiradate, S., de Jonge, L.W., Kawamoto, K., 2016, Soil-water repellency characteristic curves for soil profiles with organic carbon gradients. Geoderma, 264,150-159. 
Yanashima, R., García, A.A., Aldridge, J., Weiss, N., Hayes, M.A., Andrews, J.H., 2012, Cutting a drop of water pinned by wire loops using a superhydrophobic surface and knife. PLoS ONE 7, e45893.

Yarin, A.L., 2006, Drop impact dynamics: splashing, spreading, receding, bouncing. Annu. Rev. Fluid Mech., 38, 159-192.

Zhang, M., Feng, S., Wang, L., Zheng, Y., 2016, Lotus effect in wetting and self-cleaning. Biotribology, 5, 31-43.

Zhang, R., Farokhirad, S., Lee, T., Koplik, J., 2014, Multiscale liquid drop impact on wettable and textured surfaces. Physics of Fluids, 26, 082003.

\section{Graphical Abstract}



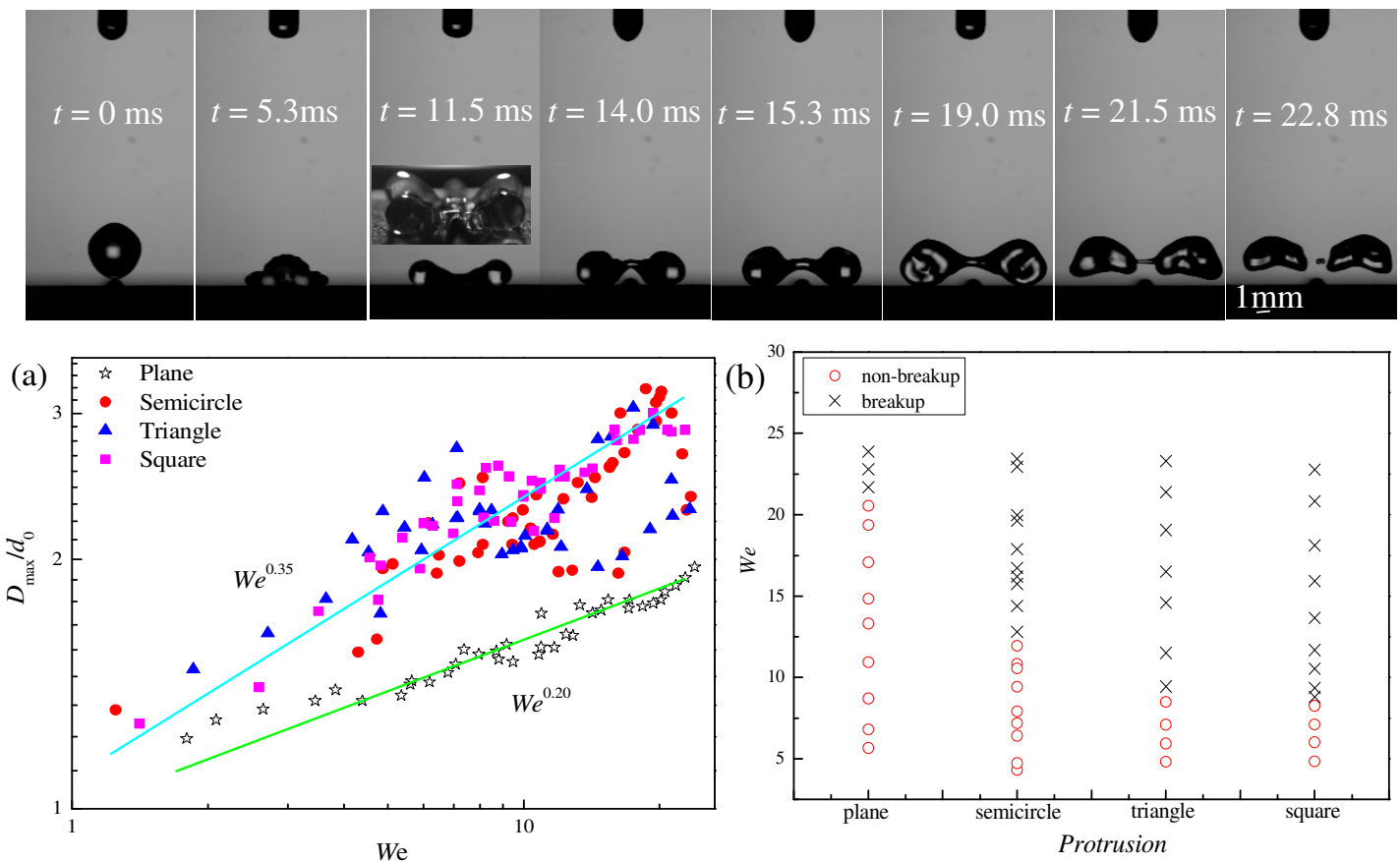

Example of a water drop impact on a protrusion of semicircle geometry and the presence of protrusions enhances the expanding and breakup stage 